\title{
The Impact on the Long-term Outcomes of Hormonal Status After Hepatic Resection for Breast Cancer Liver Metastases
}

\author{
NICOLAE BACALBASA ${ }^{1,2^{*}}$, IRINA BALESCU ${ }^{3}$, VERONICA ILIE ${ }^{2}$, RALUCA FLOREA $^{2}$, ANDREI SOROP $^{2}$, \\ VLADISLAV BRASOVEANU ${ }^{4 *}$, IULIAN BREZEAN ${ }^{1,5^{*}}$, MIHAELA VILCU $^{1,5^{*}}$, SIMONA DIMA $^{2,4}$ and IRINEL \\ POPESCU 4,6 \\ 1 "Carol Davila" University of Medicine and Pharmacy, Bucharest, Romania; \\ ${ }^{2}$ Center of Excellence in Translational Medicine - Fundeni Clinical Institute, Bucharest, Romania; \\ 3 "Ponderas" Academic Hospital, Bucharest, Romania; \\ 4 "Dan Setlacec" Center of Gastrointestinal Disease and Liver Transplantation, \\ Fundeni Clinical Institute, Bucharest, Romania; \\ 5"Ion Cantacuzino" Clinical Hospital, Bucharest, Romania; \\ 6“Titu Maiorescu” University, Bucharest, Romania
}

\begin{abstract}
Background/Aim: Breast cancer remains one of the most frequently encountered malignancies worldwide, which is in most cases diagnosed in early stages of disease. However, although surgery and adjuvant oncological treatment are performed with curative intent, a certain number of cases will develop distant metastases. In cases presenting oligometastatic disease, surgery might be tempted in order to maximize the benefit in terms of survival. The aim of this paper was to identify which cases could benefit most after liver resection for breast cancer liver metastases. Materials and Methods: The study included 67 patients submitted to surgery for breast cancer liver metastases between 2003 and 2017 in the "Dan Setlacec" Center of Gastrointestinal Diseases and Liver Transplantation, Fundeni Clinical Institute. Results: Patients diagnosed with hormone-positive breast tumors reported a significantly higher disease-free and overall survival after resection of the primary tumor. After resection for breast cancer liver metastases, patients presenting hormone receptors at the level of the metastatic sites also experienced a better outcome when
\end{abstract}

This article is freely accessible online.

*These Authors contributed equally to this work.

Correspondence to: Irina Balescu, "Ponderas" Academic Hospital, Bucharest, Romania. Tel: +40 724077709, e-mail: irina.balescu@ ponderas-ah.ro

Key Words: Breast cancer, liver metastases, hormonal receptor, HER2 status. compared to those in which hormonal receptors were absent. However, the difference was not statistically significant. Conclusion: Liver resection for breast cancer liver metastases seems to be associated with the best outcomes in terms of survival in patients presenting positive hormonal receptors status.

Breast cancer remains the most frequently diagnosed malignancy in both the Western World and United States of America, most cases being diagnosed in early stages of disease $(1,2)$. Despite this fact, up to one third of these patients will develop distant metastases at a certain point of their evolution (3). When it comes to hepatic metastases, although liver represents the third most frequent site of metastatic spread, it is estimated that only less than $25 \%$ of cases will be amenable to surgery with curative intent. This fact is mainly related to the aspect that liver metastases usually occur as part of systemic recurrence; under these conditions the patients are rather candidates for palliative oncologic treatments while the overall survival rates remain extremely poor $(3,4)$. In cases in which oligometastatic liver lesions are encountered, surgery might be taken into consideration with curative intent. In such cases the overall prognosis is significantly improved, five-year survival rates of $30-50 \%$ being reported so far $(5,6)$; however, although intraoperative techniques and postoperative management has been significantly improved, perioperative morbidity rates remain high, reaching up to $15 \%$ (6). Due to this reason a proper selection of the patients who could benefit most after this kind of surgery is mandatory. In consequence, attention was given on determining which the most important factors of prediction for long-term survival after breast cancer liver metastases are. 


\section{Materials and Methods}

The study was conducted on 67 patients diagnosed with breast cancer liver metastases between 2003 and 2017, after obtaining the approval of the Ethical Department for Clinical Trials (no 30504/17.10.2017) in "Dan Setlacec" Center of Gastrointestinal Diseases and Liver Transplantation, Fundeni Clinical Institute. The main inclusion criteria included: prior surgical treatment for the breast tumor, the evidence of unique or multiple breast cancer liver metastases, the absence of any kind of treatment (including chemotherapy, radiotherapy or immunotherapy) in the last two weeks before entering the study, a good biological status as well as the written informed consent obtained from all patients. Each patient received a numeric code in order to provide an exact identification of the case while the identity of each patient remained anonymous. In each case both blood tests as well as tissues from liver metastases were harvested. In all cases resection of the breast cancer liver metastases was performed.

Samples from liver metastases placed in paraffin were fixed in formalin solution (10\%) and therefore cut in sections of $2 \mu \mathrm{m}$ each; each section was submitted to standard hematoxylin-eosin staining. In order to perform the immunohistochemical studies, the paraffin was taken away with xylene solvent while the resulting tissues were rehydrated. After complete solving of the paraffin residues immunohistochemical studies regarding the presence of cytokeratin 6, estrogen receptors, Ki67, ERbb2/HER2 and progesterone receptors were performed, the images being achieved by using an Olimpus BX43 microscope. The standard concentrations for the antibodies which were used in order to identify these receptors are presented in Table I.

\section{Results}

The initial data base included data regarding age, general status, histopathological characteristics of the primary tumor, the type of the first intention therapy as well as the characteristics at the time of diagnosing the recurrence. The median age at the time of diagnosis for breast cancer was 52 years (range $=31-79$ years). Data regarding recurrence included the number and size of the liver lesions, the degree of differentiation, the presence of other extrahepatic metastases as well as details regarding the first intention therapy.

The pathological characteristics of the breast tumor for the 67 patients introduced in the study are presented in Table II. When it comes to the hormonal status, details regarding the estrogen and progesterone receptors were available in 38 cases; among these cases 31 patients were ER positive and 7 cases were ER negative, 30 cases were PR positive while the remaining 8 cases were PR negative. In the meantime, details regarding HER2 status were available in 37 cases (36 patients presenting negative staining for HER2 while a single case was HER2 positive).

All 67 patients were therefore submitted to surgery for breast cancer liver metastases. Data regarding the characteristics of the liver metastases from breast cancer are presented in Table III.
Table I. Standard concentrations for the antibodies that were used in order to identify the presence of cytokeratin 6, oestrogen receptors, Ki67, ERbb2/HER2 and progesterone receptors.

\begin{tabular}{lcc}
\hline Antibody & $\begin{array}{c}\text { Standard } \\
\text { concentration }\end{array}$ & $\begin{array}{c}\text { Protocol } \\
\text { concentration }\end{array}$ \\
\hline Cytokeratin 6 & $0.5 \mathrm{mg} / \mathrm{ml}$ & $10 \mu \mathrm{g} / \mathrm{ml}$ \\
Estrogen Receptors & $0.5 \mathrm{mg} / \mathrm{ml}$ & $25 \mu \mathrm{g} / \mathrm{ml}$ \\
Ki67 & $0.5 \mathrm{mg} / \mathrm{ml}$ & $0.7 \mu \mathrm{g} / \mathrm{ml}$ \\
ERbb2/HER2 & $0.5 \mathrm{mg} / \mathrm{ml}$ & $5 \mu \mathrm{g} / \mathrm{ml}$ \\
Progesterone Receptors & $0.5 \mathrm{mg} / \mathrm{ml}$ & $2.5 \mu \mathrm{g} / \mathrm{ml}$ \\
\hline
\end{tabular}

Table II. Characteristics of breast tumors for the 67 patients.

\begin{tabular}{lc}
\hline Characteristics & No of cases \\
\hline T & \\
T1-2 & 30 \\
T3-4 & 18 \\
NA & 19 \\
N & \\
N0 & 17 \\
N1 & 31 \\
NA & 19 \\
M & \\
M0 & 41 \\
M1 & 7 \\
NA & 19 \\
Grade of differentiation & \\
G1 & 6 \\
G2 & 21 \\
G3 & 14 \\
NA & 26 \\
FIGO stage & \\
IA & 7 \\
IIA-IIB & 21 \\
IIIA-IIIB & 15 \\
IV & 7 \\
NA & 17 \\
Hormonal status & \\
ER positive/negative $(\mathrm{n}=38)$ & $31 / 7$ \\
PR positive/negative $(\mathrm{n}=38)$ & $30 / 8$ \\
HER2 positive/negative $(\mathrm{n}=37)$ & $1 / 36$ \\
\hline
\end{tabular}

Immunohistochemistry studies regarding ER, PR, ERbb2/HER2, Ki67 and CK6 for metastatic lesions were available in 26 patients; among these cases, CK6 was negative in $92.6 \%$ of cases while in the remaining $7.4 \%$ of cases only $5-10 \%$ of the cells presented a positive staining for the marker. The percent of positive cells for ERb ranged between 10 and $70 \%$, PR was positive in up to $59 \%$ of cases; HER2 was negative in $70 \%$ of patients while the level of Ki67 ranged between 5 and $50 \%$.

When it comes to the long-term outcomes, the three years overall survival after breast surgery was $94 \%$ while the five years overall survival rate was $69 \%$. 
Table III. Characteristics at the time of liver resection for breast cancer liver metastases $(n=67)$.

\begin{tabular}{lc}
\hline Characteristics & No. of cases \\
\hline Number of liver metastases & 33 \\
Unique & 22 \\
Multiple & 12 \\
NA & \\
Size of the largest lesion & 42 \\
$<5$ cm & 13 \\
$>5$ cm & 12 \\
NA & \\
M & 41 \\
M0 & 7 \\
M1 & 19 \\
NA & \\
Grade of differentiation & 22 \\
G1-G2 & 29 \\
G2-G3 & 16 \\
NA & \\
Type of liver metastasis & 7 \\
Synchronous & 54 \\
Metachronous & 6 \\
NA & \\
Resection margin status & 62 \\
R0 & 5 \\
R1 &
\end{tabular}

Kaplan Meyer survival analysis performed based on ER, PR and HER2 expression in breast tumors revealed that the positivity of ER and PR respectively significantly influenced the overall survival (Figures 1 and 2) while the positivity of HER2 did not significantly affect survival (Figure 3); however, HER2 positive patients reported a three-year overall survival of $38 \%$ while the five-year survival rate among these cases was of only $14,4 \%$. When it comes to the disease-free survival, $40,7 \%$ of the HER2 patients presented no sign of recurrence at three years follow up while only $12,21 \%$ of the same group remained free of disease at a 5-year follow up; however, these data had no statistical significance $(p=0.67)$ (Figure 4$)$. In the mean-time $45,9 \%$ of patients with positive ER or PR remained free of disease at three years follow up while $25,7 \%$ of them remained free of disease at a five year follow up, both types of receptors significantly influencing the disease-free survival (Figures 5 and 6).

The same analysis was than performed in order to establish whether the presence of ER/PR/HER-2 at the level of the metastatic lesion influenced survival. However, none of the mentioned receptors influenced significantly the disease-free survival nor the overall survival after liver resection for breast cancer liver metastases (Figure 7A, B and $\mathrm{C}$ and Figure 8A, B and $\mathrm{C}$ ).

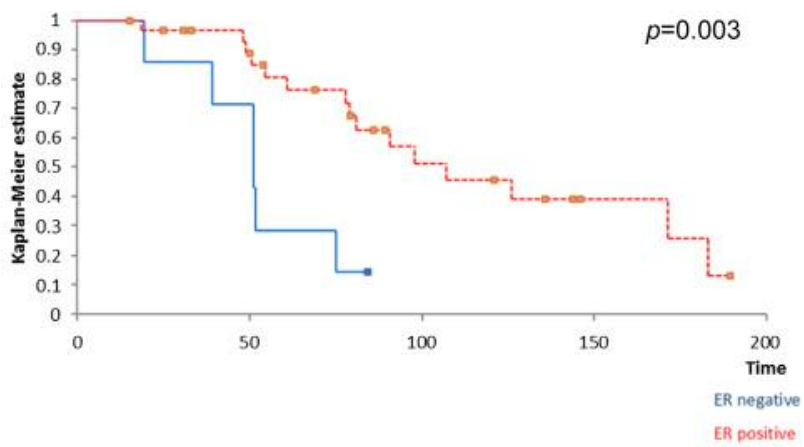

Figure 1. The influence of ER status on survival; patients with positive ER reported a significantly higher overall survival.

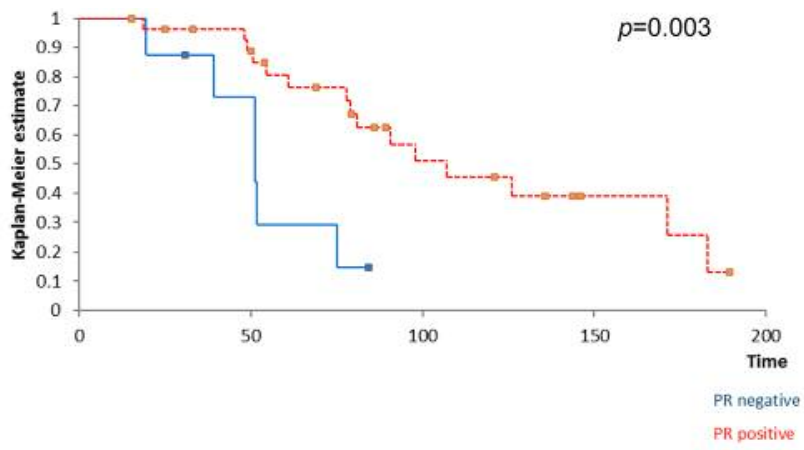

Figure 2. The influence of PR status on survival; patients with positive $P R$ reported a significantly higher overall survival.

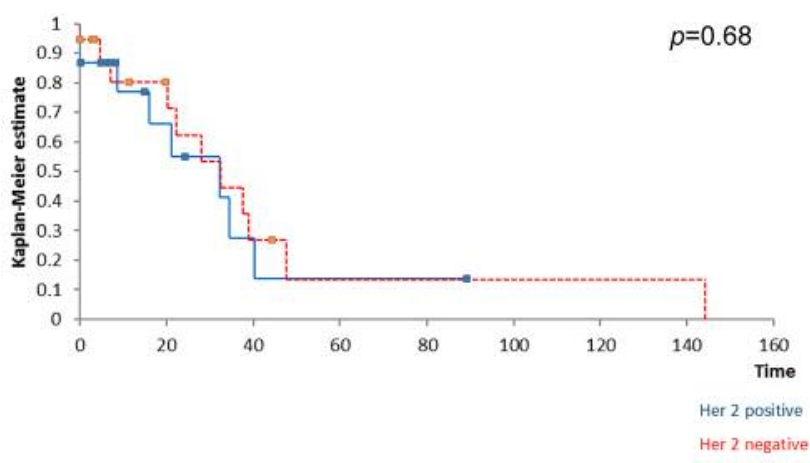

Figure 3. HER2 status did not significantly influence survival.

However, patients presenting ER/PR at the level of the breast cancer liver metastases trended to have a better outcome in terms of both disease-free and overall survival when compared to those with negative hormonal receptors. In the meantime, patients with HER2 receptors trended to have a poorer outcome in terms of both disease-free and overall survival. 


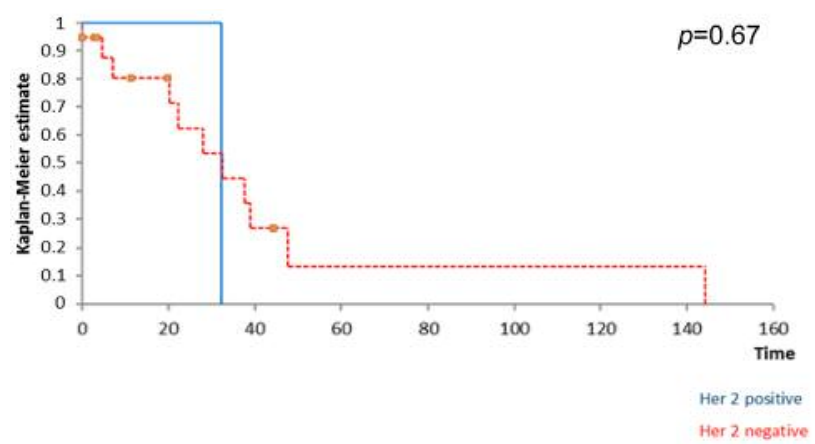

Figure 4. HER2 status did not significantly influence disease-free survival.

\section{Discussion}

Metastatic breast cancer is a systemic disease with very poor survival rates; despite recent advances, once liver metastases develop, the overall survival rates remains very low, most patients succumbing during the first years after the diagnosis of liver lesions $(7,8)$. Moreover, the efficacy of systemic therapies in such cases remains very limited especially due to the high chemoresistance as well as to the negative hormone receptor status of most hepatic metastases (9). An interesting finding in regard to the influence of ER on the pattern of spread in breast cancer was reported by Samaan and co. even from the early '80s. According to their study conducted on 262 patients diagnosed with breast cancer, the presence of ER was rather associated with skin, bone and lymph node metastases; in the meantime, patients with ER- status had a higher risk of developing visceral metastases. Among the 73 cases who developed metastatic disease ER positivity was seen in $60 \%$ of cases with skin, bone or lymph node metastases and only in $30 \%$ of cases with liver metastases (10). A more recent study conducted on the theme of correlation between the type of receptors and the risk of development of breast cancer liver metastases demonstrated that increased phosphorylation of HER2 plays a crucial role in the induction of breast cancer liver metastases development; moreover, an association between high serum levels of HER2 and lack of ER doubles the relative risk of progression of disease and cancer related mortality (11).

However, the hormonal receptor status, as well as HER2 status in metastatic lesions should be tested from the metastatic site and should not be extrapolated from the initial breast tumor due to the possibility of receptor conversion phenomenon. Therefore, once metastatic lesions from breast cancer are diagnosed, treatment options should not be tailored according to the initial receptor status of the primary tumor, a detailed analysis of these receptors from the metastatic sites being needed. One of the most interesting studies regarding receptor conversion in breast cancer distant metastases comes from

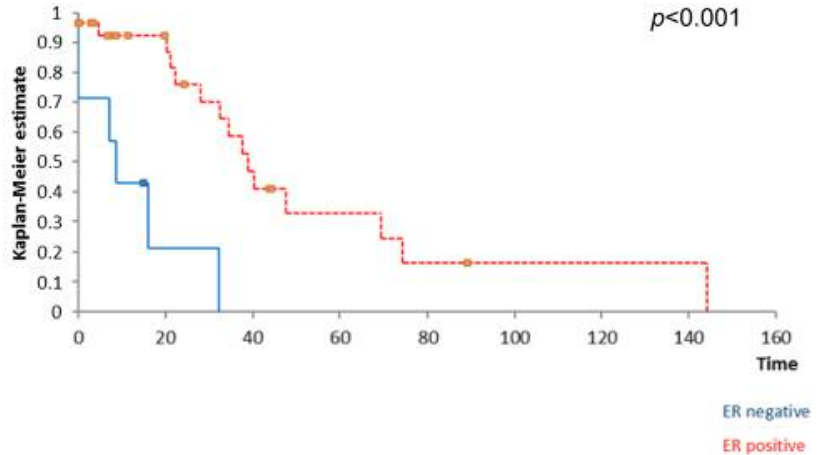

Figure 5. The influence of ER positivity on disease-free survival.

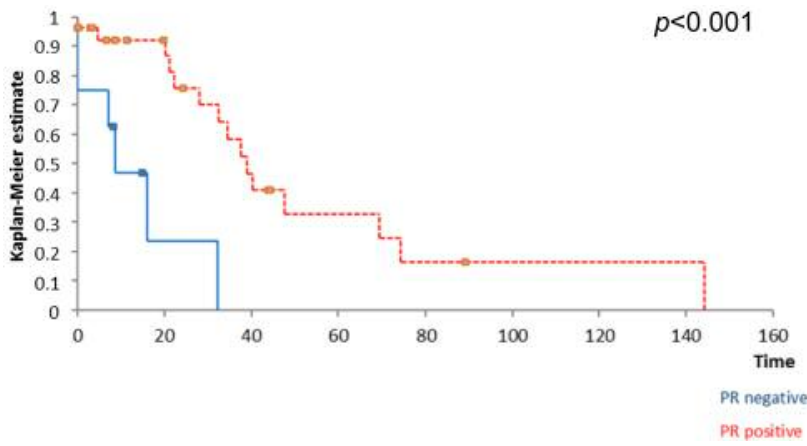

Figure 6. The influence of $P R$ positivity on disease-free survival.

Laurien Hoefnagel and was published in 2010 in Breast Cancer Research Journal (12). The study included 233 distant breast cancer metastases (from skin -76 cases, liver -63 cases, lung - 43 cases, brain - 44 cases and respectively gastrointestinal tract -7 cases). In all cases, immunohistochemical studies in order to determine the presence of ER, PR and HER2 were performed. The authors demonstrated that conversion from $\mathrm{ER}+/ \mathrm{PR}+$ to $\mathrm{ER}-/ \mathrm{PR}-$ was encountered in $12,4 \%$ of cases while an inverse conversion (from $\mathrm{ER}-/ \mathrm{PR}-$ to $\mathrm{ER}+/ \mathrm{PR}+$ ) was reported in $8,2 \%$ of cases. As for the HER2 status, conversion was seen in 5,2\% of cases. Moreover, the authors demonstrated that receptor conversion phenomenon is also influenced by the location of the metastatic site, being more frequently encountered for brain, liver and gastrointestinal metastases (12). Therefore, a specific immunohistochemical study of the metastatic lesions is mandatory in order to establish which the most efficient treatment option is.

When it comes to liver resection for breast cancer liver metastases, the most commonly reported factors that seem to influence the long-term outcomes are related to the ability of performing an R0 resection as well as to the absence of extrahepatic disease (13-18). However, it seems that the 


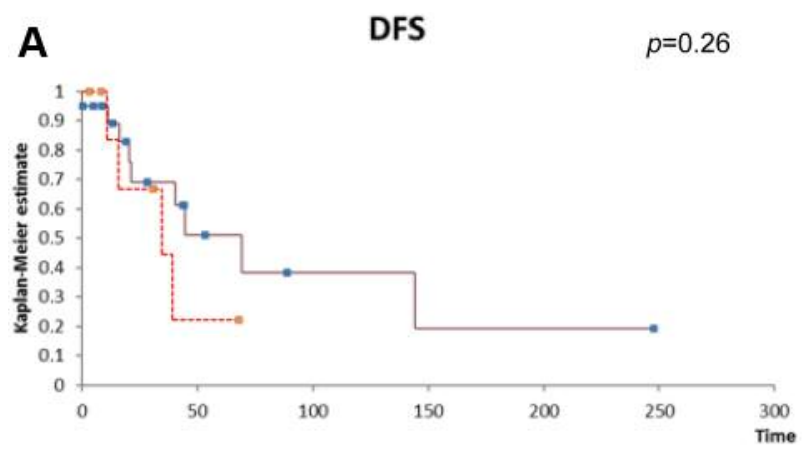

ER positive

B

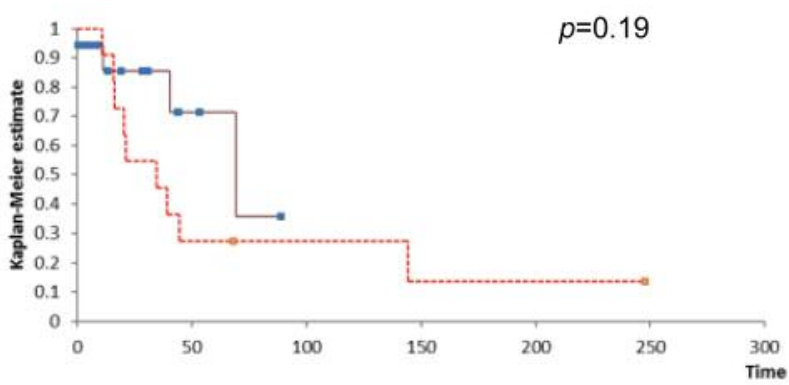

PR positive

PR. negative

C

DFS

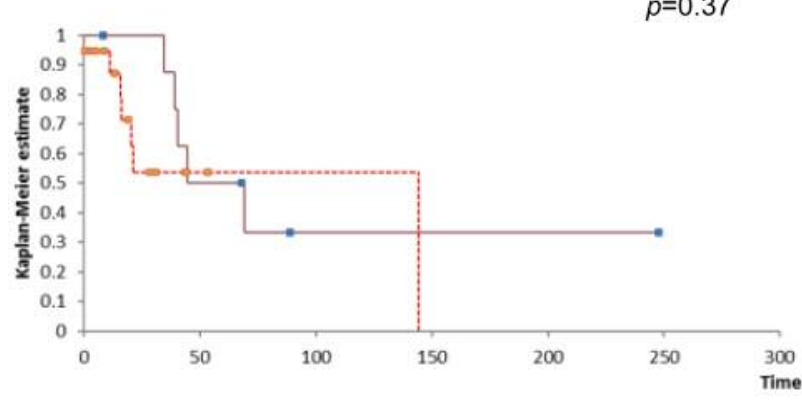

HER2 negative

HER2 positive

Figure 7. The positivity of $E R(A), P R(B)$ or Her2 $(C)$ at the level of the breast cancer liver metastases did not significantly influence the diseasefree survival after liver resection. A. The influence of the presence of ER at the level of breast cancer liver metastases on disease-free survival. $B$. The influence of the presence of $P R$ at the level of breast cancer liver metastases on disease-free survival. C. The influence of the presence of HER2 at the level of breast cancer liver metastases on disease-free survival.

presence of receptors at the level of the metastatic site might also play an important role.

The impact of hormone receptor status on the overall survival in breast cancer liver metastases was also studied by
A $\quad$ OS $\quad p=0.73$

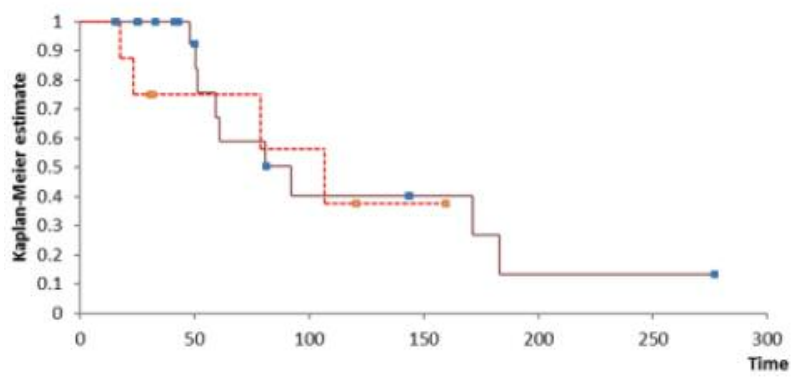

ER positive

ER negative

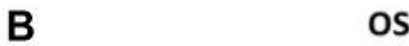

$p=0.27$

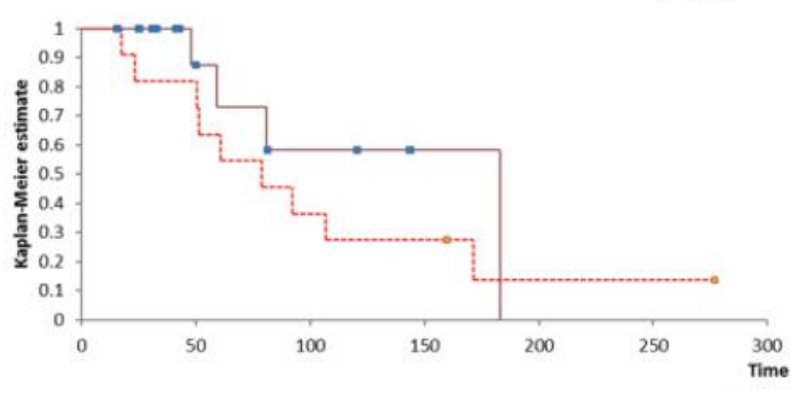

PR positive

$P$ negative
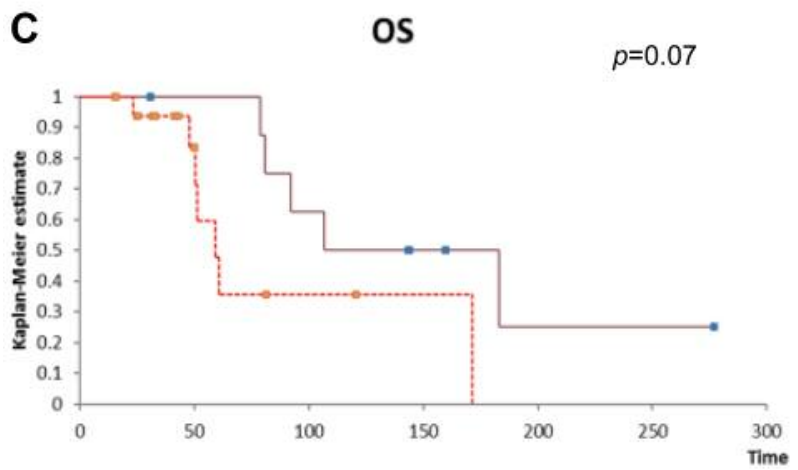

HER2 negative

HERQ positive

Figure 8. The positivity of ER (A), PR (B) or HER2 (C) at the level of the breast cancer liver metastases did not significantly influence the overall free survival after liver resection. A. The influence of the presence of ER at the level of breast cancer liver metastases on overall survival. B. The influence of the presence of $P R$ at the level of breast cancer liver metastases on overall survival. C. The influence of the presence of HER2 at the level of breast cancer liver metastases on overall survival.

Dominique Elias in the paper that was published in February 2003 in The American Journal of Surgery (19). The study included 54 breast cancer patients diagnosed with breast cancer liver metastases who were submitted to surgery 
between 1986 and 2000. The only significant prognostic factor which influenced the overall survival after liver resection for metastatic lesions was the presence of hormone receptors, the relative risk of death being increased by 3,5fold when negative; in the meantime, the postoperative morbidity was $12,9 \%$. Therefore, the authors stated that hepatic resection for breast cancer liver metastases should be addressed especially to patients presenting positive hormone receptor status; this decision should also take into consideration the patient's age, elderly cases with negative hormone receptor status being considered even a contraindication for hepatic resection (19).

An important study which demonstrated that clinical breast cancer subtype remains an important prognostic factor in order to predict the long-term outcomes was conducted by Duan et al. and was published in the Internal Journal of Clinical Oncology in 2013 (20). The study included 123 patients diagnosed with breast cancer liver metastases at Tianjin Medical University Cancer Hospital between 1995 and 2008. The main inclusion criteria consisted of surgical treatment for the primary tumor, metachronous liver metastases as the initial type of relapse and in the absence of other extra-hepatic lesions and complete clinical and follow-up information. In all cases the receptor status of ER, PR as well as HER2 were studied at the level of the primary breast tumor. According to this condition, $56 \%$ of cases were ER negative lesions, $60 \%$ of cases were PR negative lesions while $48 \%$ of patients presented HER2-negative tumors. When it comes to the most frequently performed therapeutic strategies for breast cancer liver metastases, $15 \%$ of cases underwent resection or radiofrequency ablation while the other cases benefited from transarterial chemoembolization or systemic chemotherapy; however, neither the initial status of hormonal receptors nor HER2 status influenced the therapeutic strategy for liver metastases. However, diseasefree survival between the treatment for the primary tumor and the diagnosis of liver metastases was significantly shorter in triple negative breast cancer as well as in cases with no hormonal receptor but with positive HER2 expression when compared to patients presenting positive hormonal receptors and negative HER2 status or both positive hormone receptors and positive HER2 expression. In the meantime, patients with triple negative tumors reported a significantly poorer overall survival in comparison with all the other categories of patients. Moreover, after breast cancer liver metastases resection, patients with triple-negative lesions experienced the poorest overall survival (when compared to all the other subtypes). In univariate analysis the most important prognostic factors which influenced survival after liver resection remained the receptor status, followed by the number of liver metastases, disease free survival and high histological grades. The influence of hormonal status persisted to be a significant prognostic factor also in multivariate analysis. Due to this reason the authors concluded that liver resection for breast cancer liver metastases in triple negative lesions is associated with the poorest long-term outcomes (20). In consequence, in such cases novel agents are to be expected in order to achieve a good control of the disease while surgery should be reserved especially for cases with positive hormone receptors and, in certain cases, for positive HER2 status.

\section{Conclusion}

The presence of hormonal receptors status represents one of the most important factors in regard to the long-term outcomes of patients with breast cancer. Therefore, patients presenting positive ER or PR seem to have the best longterm outcomes in terms of disease-free and overall survival; in the meantime, the HER2 status also seems to play an important role in predicting long-term outcomes. When it comes to the influence of hormone receptors at the level of breast cancer liver metastases, in our study their presence did not significantly modify survival; however, a better outcome was reported in cases presenting positive hormonal receptors status when compared to cases with negative ER or PR. In the meantime, patients presenting positive HER2 status trended to have a poorer outcome after liver resection for breast cancer liver metastases. In conclusion, the best long-term results after breast cancer liver metastases resection are to be expected in patients presenting hormonal receptors in both the primary tumor and at the level of the metastatic site. Therefore, surgery for breast cancer liver metastases should be offered particularly for this specific subset of patients. Although a similar conclusion could not be also affirmed in regard to HER2 status, it seems that a better outcome is to be expected after liver resection for breast cancer liver metastases in the absence of HER2 receptors at the level of the metastatic sites.

\section{Acknowledgements}

This work was supported by the project entitled "Multidisciplinary Consortium for Supporting the Research Skills in Diagnosing, Treating and Identifying Predictive Factors of Malignant Gynecologic Disorders", project number PN-III-P1-1.2-PCCDI2017-0833.

\section{References}

1 Spolverato G, Vitale A, Bagante F, Connolly R and Pawlik TM: Liver resection for breast cancer liver metastases: a cost-utility analysis. Ann Surg 265: 792-799, 2016.

2 Jemal A, Siegel R, Ward E, Hao Y, Xu J, Murray T and Thun MJ: Cancer statistics, 2008. CA Cancer J Clin 58: 71-96, 2008. 
3 Redig AJ and McAllister SS: Breast cancer as a systemic disease: a view of metastasis. J Intern Med 274: 113-126, 2013.

4 Insa A, Lluch A, Prosper F, Marugan I, Martinez-Agullo A and Garcia-Conde J: Prognostic factors predicting survival from first recurrence in patients with metastatic breast cancer: analysis of 439 patients. Breast Cancer Res Treat 56: 67-78, 1999.

5 Charalampoudis P, Mantas D, Sotiropoulos GC, Dimitroulis D, Kouraklis G and Markopoulos C: Surgery for liver metastases from breast cancer. Future Oncol 11: 1519-1530, 2015.

6 Margonis GA, Buettner S, Sasaki K, Kim Y, Ratti F, Russolillo N, Ferrero A, Berger N, Gamblin TC, Poultsides G, Tran T, Postlewait LM, Maithel S, Michaels AD, Bauer TW, Marques H, Barroso E, Aldrighetti L and Pawlik TM: The role of liverdirected surgery in patients with hepatic metastasis from primary breast cancer: a multi-institutional analysis. HPB (Oxford) 18: 700-705, 2016.

7 Fisher B, Jeong JH, Anderson S, Bryant J, Fisher ER and Wolmark N: Twenty-five-year follow-up of a randomized trial comparing radical mastectomy, total mastectomy, and total mastectomy followed by irradiation. N Engl J Med 347: $567-$ 575, 2002.

8 Eichbaum MH, Kaltwasser M, Bruckner T, de Rossi TM, Schneeweiss A and Sohn C: Prognostic factors for patients with liver metastases from breast cancer. Breast Cancer Res Treat 96: 53-62, 2006.

9 Hoffmann K, Franz C, Hinz U, Schirmacher P, Herfarth C, Eichbaum M, Buchler MW and Schemmer P: Liver resection for multimodal treatment of breast cancer metastases: identification of prognostic factors. Ann Surg Oncol 17: 1546$1554,2010$.

10 Samaan NA, Buzdar AU, Aldinger KA, Schultz PN, Yang KP, Romsdahl MM and Martin R: Estrogen receptor: a prognostic factor in breast cancer. Cancer 47: 554-560, 1981.

11 Jensen BV, Johansen JS and Price PA: High levels of serum HER-2/neu and YKL-40 independently reflect aggressiveness of metastatic breast cancer. Clin Cancer Res 9: 4423-4434, 2003.
12 Hoefnagel LD, van de Vijver MJ, van Slooten HJ, Wesseling P, Wesseling J, Westenend PJ, Bart J, Seldenrijk CA, Nagtegaal ID, Oudejans J, van d, V, van der GP, de Vries EG, van der WE and van Diest PJ: Receptor conversion in distant breast cancer metastases. Breast Cancer Res 12: R75, 2010.

13 O'Rourke TR, Tekkis P, Yeung S, Fawcett J, Lynch S, Strong R, Wall D, John TG, Welsh F and Rees M: Long-term results of liver resection for non-colorectal, non-neuroendocrine metastases. Ann Surg Oncol 15: 207-218, 2008.

14 Raab R, Nussbaum KT, Behrend M and Weimann A: Liver metastases of breast cancer: results of liver resection. Anticancer Res 18: 2231-2233, 1998.

15 Yoshimoto M, Tada T, Saito M, Takahashi K, Uchida Y and Kasumi F: Surgical treatment of hepatic metastases from breast cancer. Breast Cancer Res Treat 59: 177-184, 2000.

16 Harrison LE: Selecting the highly selective. Ann Surg Oncol 12: 420-421, 2005.

17 Bacalbasa N, Dima SO, Purtan-Purnichescu R, Herlea V and Popescu I: Role of surgical treatment in breast cancer liver metastases: a single center experience. Anticancer Res 34: 55635568,2014

18 Bacalbasa N, Balescu I, Dima S and Popescu I: Long-term survivors after liver resection for breast cancer liver metastases. Anticancer Res 35: 6913-6917, 2015.

19 Elias D, Maisonnette F, Druet-Cabanac M, Ouellet JF, Guinebretiere JM, Spielmann M and Delaloge S: An attempt to clarify indications for hepatectomy for liver metastases from breast cancer. Am J Surg 185: 158-164, 2003.

20 Duan XF, Dong NN, Zhang T and Li Q: The prognostic analysis of clinical breast cancer subtypes among patients with liver metastases from breast cancer. Int J Clin Oncol 18: 26-32, 2013.

Received May 8, 2018

Revised May 30, 2018

Accepted June 4, 2018 